\title{
Dental Health in Children and Adolescents with Somatic Pathology
}

\author{
Bozhbanbayeva N.
}

Atezhanov D.

Supiyev T.

\author{
Kazakh National Medical University named after S.D.Asfendiyarov, Almaty
}

\section{Doi:10.5901/mjss.2014.v5n20p2671}

\section{Abstract}

A complex clinical and instrumental evaluation is performed among 1347 children and adolescents with somatic pathology; physical development retardation is established in $61 \%$ and sexual development retardation - in $26 \%$. Among various somatic diseases, ENT-diseases (96\%), gastrointestinal (83\%), central nervous system (72\%), urogenital tract (72\%), hepatobiliary system (71\%) and other diseases were found. In children and adolescents with somatic pathology, dental diseases are occured in $40,6 \pm 0,42 \%$ cases. The most common were dental caries $(90,1 \%)$, periodontal diseases (68,3\%), and dentofacial abnormalities (41,6\%). The low hygienic status of the oral cavity was established $(H \mathrm{H}=2,4 \pm 0,28)$. Dental health in children and adolescents with somatic pathology was $59,4 \pm 0,60 \%$, which is lower than in almost normal children by $12,7 \%$ and age-related normal values by $22 \%$.

Keywords: dental diseases, dental health, somatic pathology, therapeutic and preventive measures.

\section{Introduction}

The belief on local character of dental pathology for many years was predominant. Some congenital syndromes, acute odontogenic infection, and their complications were considered as exclusions. To date, the growing evidence leads to a paradigm shift and switch to different concept, according to which evident interference between oral health and general health exists. Relationship between dental and somatic health is determined by the number of circumstances. On the one hand, oral cavity is a niche for multiple organisms being potential focuses of chronic odontogenic infection; on the other hand, the number of non-infectious diseases is characterized by oral symptoms [1]. There is a growing concern among cardiologists regarding periodontal diseases, diabetes, and cardiovascular diseases which are characterized by common risk factors, such as smoking, age, diabetes etc. Periodontitis and other dental diseases were found as risk factors for diabetes, diabetes nephropathy, and end-stage renal disease development $[2,3,4]$. Strong relationship between the pregnancy course and women's somatic and dental health was found. Studies [5] revealed that 73,9\% pregnant women required oral sanitation. In the majority women with decompensated caries an early gestational toxicosis, and in some cases, late gestational toxicosis, occurred.

Therefore, the assessment of dental status in children and adolescents, suffering from various somatic diseases, is of practical value in therapeutic and preventive activities for dental and somatic pathology.

\section{Materials and Methods}

We have observed 1347 children and adolescents with various somatic diseases in conditions of general hospital. Control group consisted of 120 children living in Almaty aged from 12 to 17 years without somatic pathology. According to WHO guidelines boys $(49,5 \%)$ and girls (50,5\%) were presented in equal numbers (12-14 years old $(n=1213), 15-17$ years old $(n=344))$. The "Dental status assessment card"(WHO 1997) is filled for all children and adolescents and included: passport data, temporomandibular joint assessment, oral mucosal status, fluorosis, enamel hypoplasia, teeth status and need for their treatment, oral hygiene, dentofacial abnormalities, periodontal index (CPITN), comorbidities and need for urgent dental care. Total 1557 Dental status assessment cards were filled with data being taken from children and adolescents with various somatic pathologies and with no somatic pathology.

Children with somatic pathology were examined by pediatrician, dentist, ENT doctor, ophthalmologist, neurologist, cardiologist, cardiosurgeon, orthopaedist, and endocrinologist. Difficult cases required consensus of experts. Children's 
and adolescents' parents were asked to give comprehensive information about child's perinatal, antenatal, and postnatal development. Parents' survey provided:

- Genealogical history taking, including parental dental diseases;

- Maternal chronic diseases: cardiovascular, endocrinological, hematological, renal, and gastrointestinal diseases;

- Maternal acute infectious diseases experienced during the pregnancy (influence, ARVI);

- Medicinal products taking during the pregnancy;

- Parental occupational hazards (physical, chemical factors, and stress);

- Parental social habits (smoking, alcohol consumption).

- Obstetrical and gynecological history: full-term pregnancy, preterm delivery, previous pregnancies outcomes;

- Pathological pregnancy and delivery: $1^{\text {st }}$ and $2^{\text {nd }}$ pregnancy halves toxicosis, threatened miscarriage, complicated delivery (abnormal delivery, fetal hypoxia, placental presentation, assisted delivery).

- Child development (Apgar score): height, body mass, feeding character, child's medical history during the first year of life.

At general examination of the child a special attention is drawn to full face and half face shape, it's vertical size proportionality, nasolabial and submental fold location, status of temporal muscles, particularly, masseter muscles. It is important to reveal developmental abnormalities and other body system (respiratory, musculoskeletal) diseases, the presence and character of social habits (lip and tongue biting, finger sucking). Muscular tone was assessed by palpation. Temporomandibular joint was palpated at articulate position, opened and closed mouth. Oral examination included assessment of status of buccal mucosa, palate, tongue, oral floor depth, tongue frenulum insertion place etc.), tongue's shape and size, dental tissue condition. Local irritants were revealed at periodontal examination: tooth feeling defects, carious and decayed teeth, dental deposits, diastemata, and tremae.

Clinical examination data were recorded into the special cards. Clinical and laboratory investigations results underwent to statistical analysis. The probability $(P)$ of studied parameters was established using the significance test with Student table. Differences were considered significant if $P<0,05[6]$.

\section{Results}

Complex clinical and laboratory examination was conducted in 1347 children and adolescents. Of them, in 821 (61\%) physical development retardation and in $350(26 \%)$ sexual development retardation were found. Among remaining 176 (13\%) children and adolescents growth and development were within normal limits. Children were complaining of malaise, weakness, skin and mucosal dryness, and frequent streptococcal impetigo. Among examined individuals various somatic diseases were found: ENT (96\%), gastrointestinal (83\%), central nervous system (72\%), urinary tract (72\%), and hepatobiliary diseases $(71 \%)$. In remaining $(40 \%-53 \%)$ persons musculoskeletal, endocrinological, cardiovascular, ocular diseases, and helminthosis were found.

Among gastrointestinal diseases gastritides and gastroduodenitides were predominant (89\%). Isolated esophagitis was diagnosed in $15 \%$ adolescents. These diseases had mostly chronic course. Among ENT diseases chronic tonsillitis was revealed in $48 \%$ and in combination with rhinitis in $43 \%$; chronic otitis was more rarely seen (12\%), mesotympanitis in $11 \%$, and conductive hearing loss is observed in $15 \%$ children. It should be noted that chronic processes in ENT organs may have aggravated course of other system chronic diseases being a source of infection with damage to other organs and systems.

Various gelmintosis types were established in $37 \%$ children and included enterobiosis and ascaridosis. One should suggest that increased gastrointestinal morbidity may be determined by descending infection (chronic odontogenous and ENT infections). These diseases may have led to development of gastrointestinal diseases, or may develop due to poorquality food consumption, inadequate chewing due to dental diseases or teeth absence, and food toxicoinfection rendering irritative action on gastric mucosa.

Among hepatobiliary system diseases in children, dyskinesia (53\%) and reactive hepatitis (36\%) were the most frequent. Urogenital diseases in all cases were presented by pyelonephritis. Among musculoskeletal system disorders impaired posture, scoliosis, pigeon thorax, flat foot were observed and combined with various dentofacial abnormalities mostly in children aged from 11 to 14 years.

Endocrinologic pathology was presented by somatic nanism (59\%) and endemic thyroid enlargement (43\%). Based on special investigations and chest X-ray, examination of cardiologist and cardiosurgeon, cardiovascular morbidity (mitral prolapse, cardiac chordal abnormalities, congenital heart disease) was established in $39 \%$ children. Among central 
nervous system disorders vertebrobasilar insufficiency (53.1\%), neurosis-like enuresis (33.5\%) and hypertensive syndrome during the puberty (465) manifesting due to hormonal dyscoordination where physical development is ahead of neuropsychic, and may lead to functional central nervous system disorders. Ocular disorders were revealed, as well: myopia, astigmatism in $44 \%$, and retinal angiopathy in $52 \%$.

Thus, in all observed children and adolescents various diseases of systems and organs were diagnosed, and dental diseases were the most prevalent. Dental caries was the most frequent dental disease and revealed in 1214 $(90,1 \pm 5,6 \%)$ observed children and adolescents with somatic pathology with cariosis process intensity $5,4 \pm 2,3$ significantly exceeding WHO guidelines (Table 1).

Table 1 - Dental caries prevalence and intensity in adolescents with various somatic diseases ( $n=1347)$

\begin{tabular}{|c|c|c|c|c|}
\hline \multirow{2}{*}{ Children and adolescents age } & \multicolumn{2}{|c|}{ Prevalence (\%) } & \multicolumn{2}{|c|}{ Intensity } \\
\cline { 2 - 4 } & $\mathrm{M}$ & $\pm \mathrm{m}$ & $\mathrm{M}$ & $\pm_{\mathrm{m}}$ \\
\hline 12 years & 99,2 & 6,5 & 4,9 & 1,9 \\
\hline 13 years & 88,5 & 5,8 & 3,6 & 2,6 \\
\hline 14 years & 76,9 & 4,7 & 6,1 & 3,2 \\
\hline 15 years & 98,7 & 6,4 & 4,7 & 2,9 \\
\hline 16 years & 82,9 & 3,9 & 5,9 & 1,7 \\
\hline 17 years & 94,5 & 5,7 & 5,2 & 3,4 \\
\hline M $m$ & 90,1 & $\pm 5,6$ & 5,4 & $\pm 2,3$ \\
\hline
\end{tabular}

Among children with dental caries subcompensated and compensated forms of the diseases prevailed. It was established that in all age-related groups the caries intensity was higher among III, IV, V health groups. Notably, from 13-years of age among these adolescents constant molar caries occurred in $98 \%$ cases. High caries activity was observed mostly in adolescents of III, IV, V health groups which was related with somatic pathology severity. The most severe caries cases were observed in children and adolescents with central nervous system pathology suffering from decompensated dental caries and pathological occlusion. Figure 1 demonstrates dentofacial region status in a patient with infantile cerebral paralysis.

In turns, teeth involved in chronic periodontitis were focuses of chronic odontogenous infection, exercised sensibilizing influence on child's health, promoted development of diseases, and aggravated course of chronic diseases in children and adolescents. Particularly, 91\% patients with chronic periodontitis had chronic gastrointestinal diseases, $43 \%$ - cardiovascular diseases, and 57\% - urogenital diseases. Non-carious dental tissue involvement revealed in $26.2 \%$ children and adolescents, more frequently it was presented by dental hyperesthesia (4\%), enamel hypoplasia (10,4\%), and phluorosis $(11,8 \%)$. These pathologies required dental restoration with tooth filling. Periodontal diseases were revealed in $920(68,3 \%)$ children and adolescents with somatic pathology. Predisposing factors were presented by unfavorable environmental factors, unhygienic oral condition, chronic internal diseases, formed dentofacial abnormalities, fixture abnormalities of labial, tongue, and soft tissues. Of periodontal diseases, the chronic catarrhal gingivitis was the most frequent (39.7\%) (Table 2).

Table 2 - Prevalence of periodontal and oral mucosal morbidity in children and adolescents with various somatic diseases $(n=1347)$

\begin{tabular}{|c|c|c|c|c|c|c|c|c|}
\hline \multirow{2}{*}{$\begin{array}{c}\text { Number of } \\
\text { patients }\end{array}$} & \multicolumn{3}{|c|}{ Periodontal diseases } & \multicolumn{5}{|c|}{ Oral mucosal diseases } \\
\cline { 2 - 9 } & $\begin{array}{c}\text { Catarrhal } \\
\text { gingivitis }\end{array}$ & $\begin{array}{c}\text { Hyperthrophic } \\
\text { gingivitis }\end{array}$ & $\begin{array}{c}\text { Localized chronic } \\
\text { periodontitis }\end{array}$ & $\begin{array}{c}\text { Chronic aphthous } \\
\text { stomatitis }\end{array}$ & Injuries & Cheilitides & $\begin{array}{c}\text { Tongue } \\
\text { diseases }\end{array}$ & $\begin{array}{c}\text { Medical } \\
\text { stomatitis }\end{array}$ \\
\hline Total & 537 & 222 & 161 & 84 & 23 & 18 & 16 & 29 \\
\hline$\%$ & 39,7 & 16,5 & 12,1 & 6,2 & 1,7 & 1,3 & 1,2 & 2,2 \\
\hline
\end{tabular}

All examined children and adolescents with somatic pathology had unsatisfactory hygienic index $(2,4 \pm 0,28)$. The Schiller-Pissarev test was positive. Mean PMA index was $21,3 \pm 0,90 \%$ which indicates mild gingival inflammation. The following cause of chronic catarrhal gingivitis on the background of somatic pathology were dental crowding and dental malalignments. In all these patients Schiller-Pissarev test was positive (Picture 2). Gingival marginal and papillary mucosa was hyperemic, edematous, pastous, bleeding at touching, dental tartar, supra- and subdental calculus. Oral mucosal diseases were revealed in 170 (12,6\%) children and adolescents with somatic pathology. Of them, the chronic 
aphtous stomatitis was the most frequent (Table 2). Oral mucosal diseases mainly characterized by chronic relapsing course. Oral mucosal diseases mainly accompanied by gastrointestinal, renal, ENT, and hematological pathology. Dentofacial abnormalities were revealed in $41.6 \%$ children and adolescents with somatic pathology. In this patient group abnormal occlusion (22.9\%), more rarely dental crowding (11.4\%), and dystopia (7.3\%) were diagnosed. $4.7 \%$ adolescents required prosthetic rehabilitation.

Dental health assessment in children and adolescents with various somatic pathologies is an indicative test and may support their relationship [7]. Dental health was evaluated by the following criteria: dental caries and its complications, non-carious dental damage, periodontal diseases, dental and jaw abnormalities, oral mucosal diseases, secondary adentia. On oral examination special attention is paid to dental, periodontal tissue, and oral mucosal status. On dental arch examination the number of filled carious teeth requiring treatment and extraction was evaluated. All dental diseases and conditions requiring therapy and prevention were classified in accordance with instruction. One or more health level indicators were established for every nosological form in accordance with disease intensity, proposed chewing dysfunction, and complexity of therapeutic interventions (Table 3).

Table 3 - Dental health children и adolescents with somatic pathology (в \%)

\begin{tabular}{|l|c|c|c|c|}
\hline Показатели & $\begin{array}{c}\text { Almost healthy children } \\
\text { and adolescents }(n=120)\end{array}$ & $\begin{array}{c}\text { Dental health } \\
\text { level }\end{array}$ & $\begin{array}{c}\text { Children and adolescents with } \\
\text { somatic pathology }(n=1347)\end{array}$ & $\begin{array}{c}\text { Dental health } \\
\text { level }\end{array}$ \\
\hline Dental caries & 61,5 & 38,5 & 90,1 & 9,9 \\
\hline Non-caries dental diseases & 18,4 & 81,6 & 26,2 & 73,8 \\
\hline Болезни пародонта & 42,5 & 57,5 & 68,3 & 31,7 \\
\hline Dental and jaw abnormalities & 38,1 & 61,9 & 41,6 & 58,4 \\
\hline Oral mucosal diseases & 3,6 & 96,4 & 12,6 & 87,4 \\
\hline Secondary adentia & 3,4 & 96,6 & 4,7 & 95,4 \\
\hline Mean values & $27,9 \pm 0,50 \%$ & $72,1 \pm 0,59 \%$ & $40,6 \pm 0,42 \%$ & $59,4 \pm 0,60 \%$ \\
\hline
\end{tabular}

${ }^{*}$ Acceptable dental health level in children is $81,4 \%$.

\section{Discussion}

Study results confirmed the relationship between somatic and dental pathology in children and adolescents. It was established that in children and adolescents with various somatic pathology dental diseases occur in $40.6 \pm 0.42 \%$ $(27.9 \pm 0.50 \%$ in almost normal individuals, $P<0,001)$, among which dental caries $(90,1 \%)$, periodontal diseases $(68,3 \%)$, dentofactial abnormalities $(41,6 \%)$ prevailed. Detection of $4.7 \%$ children with early edentia and requiring denture treatment causes concern. In children and adolescents with somatic pathology periodontal diseases were (68,3\%) following after caries. The main risk factor for dental caries and periodontal tissue involvement was insanitary oral cavity condition (hygienic index $=2,4 \pm 0,28$ ). At the same time, experience of many countries proved that the only way to change the situation is to widely implement preventive measures. This is supported by data [8] on dental screening among third class schoolchildren after 2-year program of monitored dental cleaning during their first and second years of study. Level of hygienic status in children according to Green-Vermillion was "satisfactory" and gingival index was within normal values.

Therefore, dental health in children and adolescents with somatic pathology was $59,4 \pm 0,60 \%$ and in almost normal children (comparison group) was $72,1 \pm 0,59 \%$ (acceptable normal value is $81,4 \%$ ), which is lower by $12,7 \%$ and $22 \%$, respectively. Decreased dental health in children and adolescents with somatic pathology is mainly related to high caries and periodontal morbidity prevalence. These data were considered for therapeutic and preventive measures planning in children and adolescents with somatic pathology.

\section{Conclusions}

1. The complex clinical, laboratory, and instrumental examination 347 children and adolescents with somatic pathology revealed physical retardation in $61 \%$ and sexual development retardation in $26 \%$, and in remaining $13 \%$ children and adolescent's growth and development was within normal limits. Among various somatic diseases, the ENT diseases (96\%), gastrointestinal (83\%), central nervous system (72\%), urogenital (72\%), hepatobiliary (71\%), musculoskeletal, endocrinological, cardiovascular, helmintoses, and ocular diseases prevailed. 
2. In children and adolescents with somatic pathology dental diseases occur in $40,6 \pm 0,42 \%$ cases, mostly dental caries $(90,1 \%)$, periodontal diseases $(68,3 \%)$, and dentofacial abnormalities $(41,6 \%)$ combined with low oral hygienic status $(\mathrm{HI}=2,4 \pm 0,28)$.

3. Dental health in almost healthy children was $72,1 \pm 0,59 \%$ and case of somatic pathology $59,4 \pm 0,60 \%$ (acceptable normal value $81,4 \%$ ), which is lower by $12,7 \%$ and $22 \%$, respectively.

\section{Acknowledgements}

Authors would like to express the deepest gratitude to the KazNMU Rektor Akanov A.A. for support of innovative technologies used for studies, and to the doctors in clinics and clinical and diagnostic laboratory staff of the Republic Children's Hospital "Aksai" for painstaking work during the study and treatment of children and adolescents with various somatic and dental diseases.

\section{References}

Solovyova A.M. Relationship between dental and somatic health. Institute of Dentistry (Saints-Petersburg) 2012, 2:1-2.

Siade G.D. et al. Acute phase inflammatory response to periodontal disease in the USA population. I. Dent. Res. 2000;57:79-149.

Shultis W.A., Well E.J., Looker H.C. et al. Effect of periodontitis on overt nephropathy and end-stage renal disease in type 2 diabetes. Diabetes Care 2007;30:306-311.

Supiyev T.K., Muldasheva A.G., Zhumabayeva K.Zh. Oral cavity in children with CRF on hemodialysis. Dental issues (Almaty) 2009;12:49-50.

Suntsov V.G., Voloshin I.M. Pregnancy course, and women's somatic, and dental health. Preventive Medicine 2011;3:50-51.

Gubler E.V. Non-parametrical statistical criteria use in biomedical studies. L.: Medicine 1983:268 p.

Leus P.A. Dental health: Guidelines on assessment method, 1989:38 p.

Leus P.A., Zhugina L.F., Omelchenko Efficacy of the Program of monitored dental cleaning in stable oral hygienic habits development in primary school-aged children. Dentistry Concepts (Almaty)2013;2:11-14.

\section{Figures, Plates and Legends}

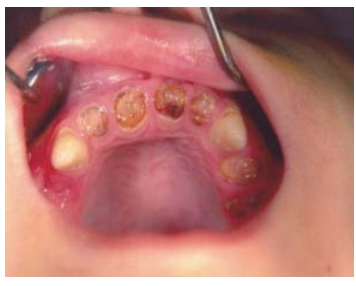

A

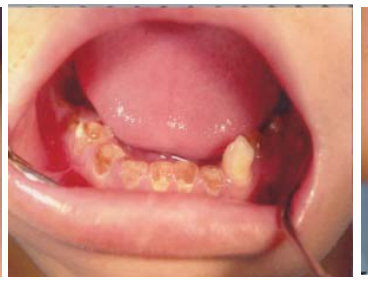

B

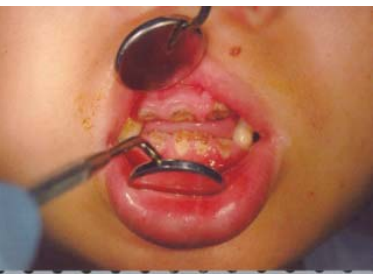

C

Graph 1 - Dental status in 14-years old patient with infantile cerebral paralysis: a) maxilla, B) mandibula, c) occlusion status.

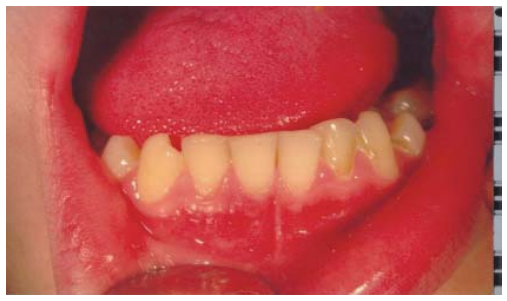

Graph 2 - Chronic catarrhal gingivitis, moderate degree, in 13 year old patients with gastrointestinal pathology. 